\title{
A mathematical model of infectious disease transmission
}

\author{
Aurelia Florea $^{1, *}$ and Cristian Lăzureanu ${ }^{2, * *}$ \\ ${ }^{1}$ Department of Applied Mathematics, University of Craiova, Romania, \\ ${ }^{2}$ Department of Mathematics, Politehnica University of Timişoara, Romania
}

\begin{abstract}
In this paper we consider a three-dimensional nonlinear system which models the dynamics of a population during an epidemic disease. The considered model is a SIS-type system in which a recovered individual automatically becomes a susceptible one. We take into account the births and deaths, and we also consider that susceptible individuals are divided into two groups: non-vaccinated and vaccinated. In addition, we assume a medical scenario in which vaccinated people take a special measure to quarantine their newborns. We study the stability of the considered system. Numerical simulations point out the behavior of the considered population.
\end{abstract}

\section{Introduction}

Concerns for communicable diseases, such as tuberculosis, malaria, and influenza, have existed since ancient times, such as the writings of Aristotle (384 BC-322 BC). In [3] the authors state that the existence of microorganisms was demonstrated by van Leeuwenhoek (16321723). The germ theory of disease, introduced by Jacob Henle (1809-1885), was developed by Robert Koch (1843-1910), Louis Pasteur (1822-1875), and Joseph Lister (1827-1912) in the latter part of the nineteenth century and the early part of the twentieth century. The first contributions in modern mathematical epidemiology were attributed to P.D. En'ko between 1873 and 1894 (see [6, 7]). Between 1900 and 1935, in a lot of books and articles, such as [11-13], A.G. McKendrick and W.O. Kermack have introduced the foundations of the entire approach to epidemiology based on compartmental models.

The recent SARS epidemic, recurring influenza pandemics, and outbursts of diseases such as the Ebola virus concern many people. On the other hand, every year, millions of people die of measles, respiratory infections and other diseases that are treated as unimportant and not considered dangerous. For most diseases, the mechanism of transmission of infections is now better known. Diseases like influenza, measles, rubella, are transmitted by viral agents, and they offer a powerful immunity against reinfection, but diseases transmitted by bacteria, like tuberculosis and meningitis, confer no immunity against reinfection. Moreover, there are infections transmitted by insects. However, a major problem remains the understanding of the mechanisms that influence the spread of certain diseases.

Mathematical modeling in epidemiology tries to predict the dynamics of the spread of disease, and it suggests control strategies. An introduction to mathematical modeling is found

\footnotetext{
*e-mail: aurelia.florea@ucv.ro

**e-mail: cristian.lazureanu@upt.ro
} 
in $[1,5]$. Many models were proposed, such as SIR, SIRS, SEIR, SEIRS, and they are improved in many recent papers (see, e.g. $[2,9,10,15]$ ).

One of the simplest models for epidemic disease is the SIR model. In this model births and deaths are omitted because the time scale of an epidemic is generally much shorter than the demographic time scale. However, there are diseases that are endemic in many parts of the world and that causes millions of deaths each year. Moreover, the presence or absence of protective immunity due to infection or vaccination will affect future transmission and illness severity. In the three-dimensional proposed model, we take into account the births and deaths, and we consider that the protective immunity is absent. Thus, we obtain a SIS-type model in which a recovered individual automatically becomes a susceptible one. We also consider that susceptible individuals are divided into two groups: non-vaccinated and vaccinated. In addition, we assume a medical scenario in which vaccinated people take a special measure to quarantine their newborns, to avoid infection with the new virus.

The paper is organized as follows. In Section 2, we present the three-dimensional considered model. In Section 3, we study the stability of this system. In Section 4, we perform some numerical simulations and we notice some conclusions.

\section{The 3D model}

In this section, we consider a SIS-type model (susceptible-infected-susceptible), which intends to describe the dynamics of an infection within the population as a function of time.

We consider that a part of people has been vaccinated for diseases that have created epidemics in the past, such as measles, tuberculosis, etc., and another segment of people has not followed the vaccination schedule. In the case of a respiratory disease generated by a new virus for which there is currently no available vaccine, the questions that are raised are whether or not the vaccinated population has (partial) immunity against it and if this population can fight more easily with the new rapidly spreading disease.

At a moment $t_{0}=0$, the analyzed population is divided into three groups: non-vaccinated susceptible individuals, vaccinated susceptible individuals, and infected individuals. At this moment, we assume that vaccinated people take a special measure to quarantine their newborns, to avoid infection with the new virus, that is only the newborns which appear in first group are considered in the following. At a moment $t \geq t_{0}$, we denote by $x(t)$ the size of the group 1 (susceptible individuals who are not vaccinated) and by $y(t)$ the size of the group 2 (susceptible individuals who are vaccinated). Both groups contain only non-infected individuals at time $t$. In addition, the size of the group of individuals infected at time $t$ (group 3 ) is $z(t)$. An individual who belongs to the third group may or may not be vaccinated. Such an individual has the disease and can transmit it. We assume that a recovered individual from the group 3 automatically becomes a susceptible one. Clearly, a non-vaccinated recovered individual will belong to the group 1, while a vaccinated recovered individual will belong to the group 2. We also consider that all vaccines were made before the initial moment of analysis, thus a new born, which is not subject of a special measure, will belong to the group 1. The gain in the group 3 is at rates proportional to the number of infected individuals from group 3 and susceptible individuals from group 1 and 2, respectively. Then, the dynamics of the interactions between the three groups is governed by the following laws:

$$
\begin{aligned}
& \dot{x}=a_{1} x-a_{2} x+a_{3} z-a_{4} x z \\
& \dot{y}=-b_{2} y+b_{3} z-b_{4} y z \\
& \dot{z}=-d_{1} z-a_{3} z-b_{3} z+a_{4} x z+b_{4} y z
\end{aligned}
$$

where: 
$a_{1}$ is the birth rate of the individuals in group 1 ,

$a_{2}$ is the death rate by any reason of the individuals in group 1 ,

$a_{3}$ is the removal rate of the infected individuals from group 3 who are not vaccinated,

$a_{4}$ is the infection rate of the individuals from group 1 ,

$b_{2}$ is the death rate by any reason of the individuals in group 2

$b_{3}$ is the removal rate of the infected individuals from group 3 who are vaccinated

$b_{4}$ is the infection rate of the individuals from group 2

$d_{1}$ is the death rate by any reason of individuals from group 3 .

If we denote

$$
a=a_{1}-a_{2}, a_{3}=b, a_{4}=c, b_{2}=m, b_{3}=n, b_{4}=p, d=d_{1}+a_{3}+b_{3},
$$

then the considered dynamics becomes

$$
\begin{aligned}
& \dot{x}=a x+b z-c x z, \\
& \dot{y}=-m y+n z-p y z, \\
& \dot{z}=-d z+c x z+p y z,
\end{aligned}
$$

where $a$ is the natural growth rate of the individuals in group $1, a \in \mathbb{R}, b, c \geq 0, m>0$, $n, p \geq 0$, and $d=d_{1}+b+n$ is the removal rate of the infected individuals, where $d_{1}>0$, thus $d>b+n$.

\section{Stability of the considered model}

In this section we find out the equilibrium points of the SIS-type system (3), and we study their stability using First Lyapunov's Stability Criterion [14] and Routh-Hurwitz Theorem [8].

The biological-feasible region of model (3) requires $x(t), y(t), z(t) \in \mathbb{R}_{+}^{3}$, for any $t \in$ $\left(t_{0}, \infty\right)$. Therefore, we take into consideration those equilibrium points $\left(x^{*}, y^{*}, z^{*}\right)$ of system (3) that satisfy the conditions $x^{*} \geq 0, y^{*} \geq 0, z^{*} \geq 0$.

It is obvious that $O(0,0,0)$ is an equilibrium point of system (3) and other equilibrium points may appear. More precisely, we have the following result.

Proposition 3.1 Let $a \in \mathbb{R}, b, c, d, m, n, p \in \mathbb{R}_{+}$, such that $d>b+n, m>0$.

Then $O(0,0,0)$ is an equilibrium point of system (3). Moreover, other equilibrium points are given by:

$\left.a_{1}\right) E_{1}^{M}(M, 0,0), M \geq 0$, if $a=0$;

$\left.a_{2}\right) E_{2}\left(\frac{d}{c}, \frac{a d n}{c m(d-b)}, \frac{a d}{c(d-b)}\right)$, if $a, c>0, p=0$;

$\left.a_{3}\right) E_{3}\left(\frac{d}{c}, 0, \frac{a d}{c(d-b)}\right)$, if $a, c, p>0, n=0$;

$\left.a_{4}\right) E_{4}\left(\frac{c d m+a d p-a n p}{c(c m+a p)}, \frac{a n}{c m+a p}, \frac{a}{c}\right)$, if $a, c, p, n>0, b=0$;

$\left.a_{5}\right) E_{5}\left(x_{5}, y_{5}, z_{5}\right)$, where

$$
x_{5}=\frac{a d p+c d m+b c m-a n p+\sqrt{\Delta}}{2 c(c m+a p)}, y_{5}=\frac{d-c x_{5}}{p}, z_{5}=\frac{d m-(c m+a p) x_{5}}{(b-d+n) p},
$$

where $\Delta=c^{2}(b-d)^{2} m^{2}-2 a c p\left(b d+b n+d n-d^{2}\right) m+(a d p-a n p)^{2}$, if $a, b, c, n, p>0$. 
Proof. In order to find the equilibrium points, we need to solve the system

$$
\left\{\begin{array}{l}
a x+b z-c x z=0 \\
-m y+n z-p y z=0 \\
-d z+c x z+p y z=0 \\
x, y, z \geq 0
\end{array},\right.
$$

under the conditions $a \in \mathbb{R}, b, c, n, p \geq 0, m>0, d>b+n$.

$a_{1}$ ) Let $a=0$. If $z=0$, then $m y=0$, that is $y=0$. We obtain a family of equilibrium points, namely $E_{1}^{M}=(M, 0,0), M \geq 0$. If $z>0$, then $c x=b$ and $-m y=(d-b-n) z$, which is false.

$a_{2}$ ) Let $a \neq 0$ and $p=0$. System (4) becomes

$$
a x+z(b-c x)=0, m y-n z=0, z(c x-d)=0 .
$$

We obtain the equilibrium point $O(0,0,0)$ and, in addition, $E_{2}\left(\frac{d}{c}, \frac{a d n}{c m(d-b)}, \frac{a d}{c(d-b)}\right)$, if $a, c>0$.

$a_{3}$ ) Let $a \neq 0, p>0$, and $n=0$. System (4) reads

$$
a x+z(b-c x)=0, y(m+p z)=0, z(c x+p y-d)=0 .
$$

Since $m>0$ and $p z \geq 0$, it follows $y=0$. Thus we obtain the equilibrium points $O(0,0,0)$ and $E_{3}\left(\frac{d}{c}, 0, \frac{a d}{c(d-b)}\right)$, if $a, c>0$.

$a_{4}$ ) Let $a \neq 0, n, p>0$ and $b=0$. System (4) takes the form

$$
x(a-c z)=0,-m y+n z-p y z=0, z(c x+p y-d)=0 .
$$

If $z=0$, then we get $x=0$ and $y=0$, that is $O(0,0,0)$. If $z \neq 0$, but $x=0$, it follows $p y=d$ and $m y=(n-d) z$, which is false. Hence $x \neq 0$. Then we obtain the equilibrium point $E_{4}\left(\frac{c d m+a d p-a n p}{c(c m+a p)}, \frac{a n}{c m+a p}, \frac{a}{c}\right)$, if $a, c>0$.

$a_{5}$ ) Let $a \neq 0, b, n, p>0$. If $z=0$, then we obtain the equilibrium point $O(0,0,0)$.

If $z>0$, then the third equation of (4) leads to $y=\frac{d-c x}{p}$, with $c x \leq d$. Thus the second equation of (4) reads $-m y+n z-d z+c x z=0$, and, adding the first equation, we get $z(d-b-n)=a x-m y$. By hypothesis, $d>b+n$, hence $a x-m y>0$, that is $a>0$. Replacing $y$, it follows $\frac{d m}{c m+a p}<x \leq \frac{d}{c}$. Moreover, $z=\frac{a x-m y}{d-b-n}=\frac{d m-(c m+a p) x}{(b-d+n) p}$.

We replace $z$ in the first equation of (4), and we find that $x$ satisfies the equation

$$
r_{2} x^{2}-r_{1} x+b d m=0, \frac{d m}{c m+a p}<x \leq \frac{d}{c},
$$

where $r_{1}=a d p+c d m+b c m-a n p$ and $r_{2}=c(c m+a p)$.

If we consider $c=0$, then the first equation of (4) becomes $a x+b z=0$, that is $x=z=0$, which is false. Hence $c>0$ and consequently $r_{2}>0$.

The discriminant of equation (5) is

$$
\Delta=c^{2}(b-d)^{2} m^{2}-2 a c p\left(b d+b n+d n-d^{2}\right) m+(a d p-a n p)^{2} .
$$

Consider the equation $\Delta=0$ with the unknown variable $m$. Its discriminant is given by $\Delta_{m}=16 a^{2} b c^{2} d n p^{2}(n+b-d)<0$. Therefore $\Delta>0$ and equation (5) has two real roots, 
$x_{1,2}=\frac{r_{1} \pm \sqrt{\Delta}}{2 r_{2}}$, which are positive. We have $x_{1}=\frac{r_{1}-\sqrt{\Delta}}{2 r_{2}}<\frac{d m}{c m+a p}$, thus this root does not correspond. We also obtain that $x_{2}=\frac{r_{1}+\sqrt{\Delta}}{2 r_{2}}$ fulfills (5). Therefore, the system has the equilibrium point

$$
E_{5}\left(x_{5}, y_{5}, z_{5}\right), x_{5}=\frac{r_{1}+\sqrt{\Delta}}{2 r_{2}}, y_{5}=\frac{d-c x_{5}}{p}, z_{5}=\frac{d m-(c m+a p) x_{5}}{(b-d+n) p},
$$

if $a, c>0$.

In the sequel, we study the stability of the equilibrium points of system (3) Denote

$$
G_{1}(x, y, z)=a x+b z-c x z, G_{2}(x, y, z)=-m y+n z-p y z, G_{3}(x, y, z)=-d z+c x z+p y z .
$$

The Jacobian matrix of system (3) is given by

$$
J(x, y, z)=\left(\begin{array}{lll}
\frac{\partial G_{1}}{\partial x} & \frac{\partial G_{1}}{\partial y} & \frac{\partial G_{1}}{\partial z} \\
\frac{\partial G_{2}}{\partial x} & \frac{\partial G_{2}}{\partial y} & \frac{\partial G_{2}}{\partial z} \\
\frac{\partial G_{3}}{\partial x} & \frac{\partial G_{3}}{\partial y} & \frac{\partial G_{3}}{\partial z}
\end{array}\right)=\left(\begin{array}{ccc}
a-c z & 0 & b-c x \\
0 & -m-p z & n-p y \\
c z & p z & c x-d+p y
\end{array}\right)
$$

Proposition 3.2 Let $a \in \mathbb{R} \backslash\{0\}, d, m>0$.

$a_{1}$ ) If $a<0$, then $O(0,0,0)$ is an asymptotically stable node of system (3).

$\left.a_{2}\right)$ If $a>0$, then $O(0,0,0)$ is an unstable saddle.

Proof. The eigenvalues of $J(0,0,0)$ are $\lambda_{1}=a, \lambda_{2}=-m<0, \lambda_{3}=-d<0$. By First Lyapunov's Stability Criterion, the conclusions immediately follow.

Remark 3.1 On one hand, if the natural growth rate a is less than zero, then the population extincts and consequently the equilibrium point $O(0,0,0)$ is an attractor. On the other hand, if $a>0$, then $O$ is unstable and thus various scenarios may appear.

Proposition 3.3 Let $p=0, a>0, c>0, b, d, m, n \in \mathbb{R}_{+}, d>b+n, m>0$.

Then $E_{2}\left(\frac{d}{c}, \frac{a d n}{c m(d-b)}, \frac{a d}{c(d-b)}\right)$ is an asymptotically stable equilibrium point of system (3). More precisely, if $a^{2} b^{2}-4 a d(b-d)^{2} \geq 0$, then $E_{2}$ is a node, else it is a focus-node.

Proof. The characteristic polynomial of $J\left(E_{2}\right)$ is given by

$$
P(\lambda)=(\lambda+m)\left(\lambda^{2}-\frac{a b}{b-d} \lambda+a d\right) .
$$

Then $\lambda_{1}=-m<0$ and $\lambda_{2,3}$ are reals negative numbers if $a^{2} b^{2}-4 a d(b-d)^{2} \geq 0$, or complex conjugate numbers with negative real part otherwise. Therefore $E_{2}$ is asymptotically stable node or an asymptotically stable focus-node.

Proposition 3.4 Let $n=0, p>0, a>0, c>0, b, d, m \in \mathbb{R}_{+}, d>b+n, m>0$.

Then $E_{3}\left(\frac{d}{c}, 0, \frac{a d}{c(d-b)}\right)$ is an asymptotically stable equilibrium point of system (3). More precisely, if $a^{2} b^{2}-4 a d(b-d)^{2} \geq 0$, then $E_{3}$ is a node, else it is a focus-node.

Proof. Taking into account that the characteristic polynomial of $J\left(E_{3}\right)$ is given by

$$
P(\lambda)=\left(\lambda+m+\frac{a d p}{c(d-b)}\right)\left(\lambda^{2}-\frac{a b}{b-d} \lambda+a d\right),
$$

the conclusion follows. 
Remark 3.2 A particular case arise whether the infection rate of the vaccinated individuals is zero. This situation means that does not exist infected vaccinated individuals and, consequently, we may consider that the removal rate $n$ of the vaccinated individuals is zero. In the hypothesis of Proposition 3.3, a local attractor $E_{2}$ appears in the considered dynamics, which becomes $E_{3}$ (see Proposition 3.4). In time, the number of vaccinated individuals vanish, because the newborns of this group are not considered in our dynamics.

Proposition 3.5 Let $b=0, p>0, a>0, c>0, n>0, d, m \in \mathbb{R}_{+}, d>b+n, m>0$.

Then $E_{4}\left(\frac{c d m+a d p-a n p}{c(c m+a p)}, \frac{a n}{c m+a p}, \frac{a}{c}\right)$ is an unstable equilibrium point of system (3).

Proof. The characteristic polynomial of $J\left(E_{4}\right)$ has the form

$$
P(\lambda)=\lambda^{3}+a_{1} \lambda^{2}+a_{2} \lambda+a_{3},
$$

where $a_{1}=\frac{c m+a p}{c}, a_{2}=\frac{a(c d m+(a d-(a+m) n) p)}{c m+a p}, a_{3}=\frac{a(c d m+a(d-n) p)}{c}$. Then $a_{1} a_{2}-a_{3}=-\frac{a m n p}{c}<0$, hence by Routh-Hurwitz Theorem the conclusion follows.

\section{Numerical simulations}

Numerical computations allow us to establish the stability of the equilibrium points. In the case $a, b, c, n, p>0$, the analytical study of the stability of the equilibrium point $E_{5}$ (see Proposition 3.1) is very difficult, if not impossible. Obviously, if we fix the values of these parameters, then we may numerically compute the coordinates of $E_{5}$ and the eigenvalues of $J\left(E_{5}\right)$. In addition, numerical simulations of the time series of the solutions $x(t), y(t), z(t)$ may be drawn.

In order to compare the behavior of the vaccinated group versus the non-vaccinated one, we firstly fix the values of all parameters except $b$ (the removal rate of the infected individuals who are not vaccinate), $n$ (the removal rate of the infected individuals who are vaccinate), and $d$, which depends on $b$ and $n$, and consider two cases: $b=n$ and $b<n$. The simulations are shown in Figure 1. We notice that the number of individuals that belong to group 2 (vaccinated, $y(t)$ ) decreases more slowly as the parameter $n$ increases, as expected. Moreover, the number of the infected people grows slowly and, consequently, the number of individuals in group 1 decreases more slowly.
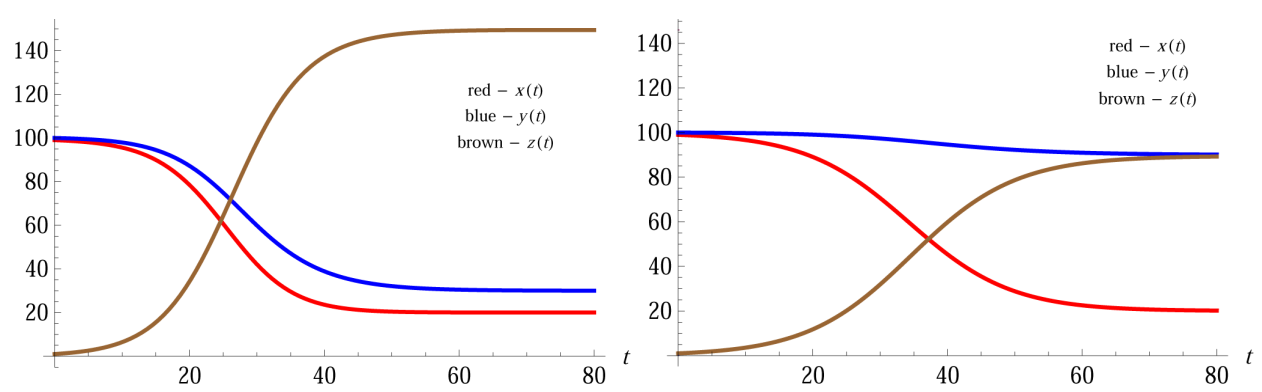

Figure 1. Time series of system (3): $n=b$ vs. $n>b\left(a=2.73973 \cdot 10^{-6}, c=0.0015, m=0.0000328767, p=0.001\right.$; left: $b=n=0.03, d=0.0600466$, right: $b=0.03, n=0.09, d=0.120047$; initial conditions: $x_{0}=99 ; y_{0}=$ $\left.100 ; z_{0}=1\right)$. 

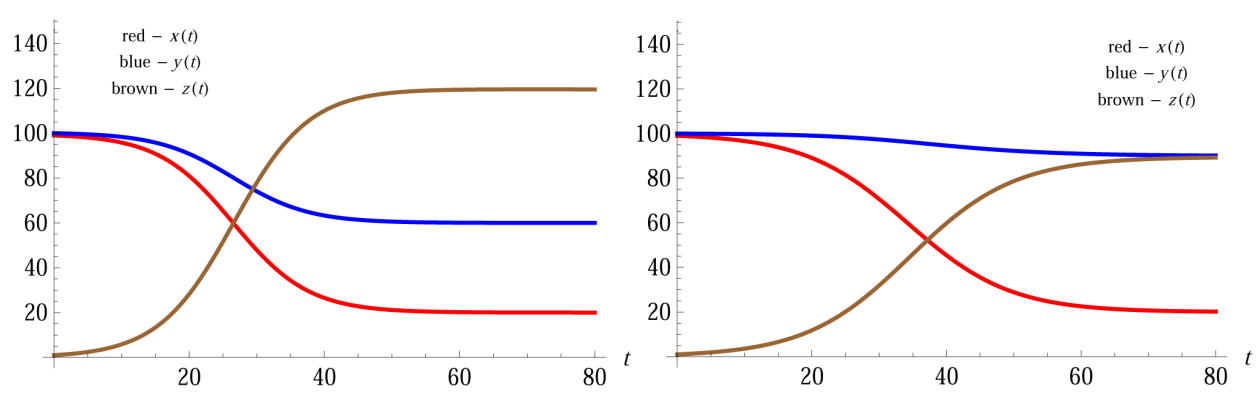

Figure 2. Time series of system (3): $p=c$ vs. $p<c\left(a=2.73973 \cdot 10^{-6}, b=0.03, c=0.0015, d=0.120047, m=\right.$ $0.0000328767, n=0.09$; left: $p=c=0.0015$, right: $p=0.001$; initial conditions: $x_{0}=99 ; y_{0}=100 ; z_{0}=1$ ).

Now, we fix the values of all parameters except $p$ (the infection rate of the vaccinated individuals), and we compare the case when $p=c$, where $c$ is the the infection rate of the non-vaccinated individuals, with the case $p<c$. The simulations are shown in Figure 2. We notice that the number of individuals that belong to group 2 (vaccinated, $y(t)$ ) decreases more slowly as the infection rate $p$ of the vaccinated individuals drops. In addition, the number of infected individuals increases more slowly.

A particular situation arises when $b=0$, that is the recovery rate of the non-vaccinated individuals vanishes. In this case, the gain in the group 1 is given only by the natural growth rate and the infected individuals that belong to this group do not recover. The simulation given in Figure 3 (right) shows that this group is extinct after a short time, which is explained by the fact that the new born are non-vaccinated, then they get infected and do not recover.
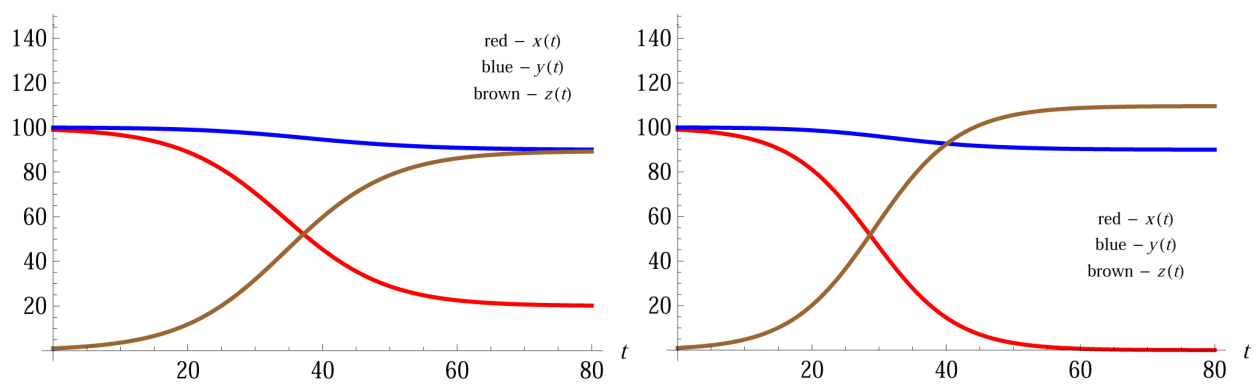

Figure 3. Time series of system (3): $b \neq 0$ vs. $b=0\left(a=2.73973 \cdot 10^{-6}, c=0.0015, m=0.0000328767, n=\right.$ $0.09, p=0.001$; left: $b=0.03, d=0.120047$; right: $b=0, d=0.0900362$; initial conditions: $x_{0}=99 ; y_{0}=$ $\left.100 ; z_{0}=1\right)$.

The above simulations suggest that the dynamics of individuals in the considered SIStype model is in concordance with the variation of the corresponding infection and recovered rates, respectively.

\section{Acknowledgments}

We would like to thank the referees very much for their valuable comments and suggestions.

This research was supported by Horizon 2020-2017-RISE-777911 project. 


\section{References}

[1] R. M. Anderson and R. M. May, Infectious Diseases of Humans (Oxford Science Publications, Oxford, 1991) 768 pp.

[2] O. N. Bjornstad, K. Shea, M. Krzywinski, et al., Nature Methods 17, 557-558 (2020).

[3] F. Brauer, and C. Castillo-Chavez, Mathematical Models in Population Biology and Epidemiology (Springer, Berlin Heidelberg New York, 2001) 440 pp.

[4] C. Castillo-Chavez, S. Blower, P. van den Driessche, D. Kirschner, and A.-A. Yakubu, Mathematical Approaches for Emerging and Reemerging Infectious Diseases: An Introduction (Springer, Berlin Heidelberg New York, 2002) 378 pp.

[5] O. Diekmann, J. A. P. Heesterbeek, and J. A. J. Metz, Journal of Mathematical Biology 28, 365-382 (1990).

[6] K. Dietz, Australian Journal of Statistics 30, 56-65 (1988).

[7] K. Dietz and K. P. Hadeler, Journal of Mathematical Biology 26, 1-25 (1988).

[8] F. R. Gantmacher, Matrix Theory, Vol. II (Chelsea Pub. Co., New York, 2000) 276 pp.

[9] S. He Y. Peng, and K. Sun, Nonlinear Dynamics 101, 1667-1680 (2020).

[10] O. Khyar and K. Allali, Nonlinear Dynamics 102, 489-509 (2020).

[11] W. O. Kermack and A. G. McKendrick, Proceedings of the Royal Society of London 115, 700-721 (1927).

[12] W. O. Kermack and A. G. McKendrick, Proceedings of the Royal Society of London 138, 55-83 (1932).

[13] W. O. Kermack and A. G. McKendrick, Proceedings of the Royal Society of London 41, 94-112 (1933).

[14] A. M. Lyapunov, Problème générale de la stabilité du mouvement, Vol. 17, (Princeton University Press, Princeton, NJ, USA, 1949) 474 pp.

[15] G. Rohith and K. B. Devika, Nonlinear Dynamics 101, 2013-2026 (2020). 